\title{
A phase III study on neoadjuvant chemotherapy versus neoadjuvant toripalimab plus chemotherapy for locally advanced esophageal squamous cell carcinoma: Henan Cancer Hospital Thoracic Oncology Group 1909 (HCHTOG1909)
}

\author{
Yan Zheng^, Xian-Ben Liu, Hai-Bo Sun, Jinliang Xu, Sining Shen, Yu-Feng Ba, Ming Yan, \\ Zimin Qin, Bao-Xing Liu, Zong-Fei Wang, Shi-Lei Liu, Rui-Xiang Zhang, Pei-Nan Chen, \\ Guang-Hui Liang, Dongfeng Yuan, Zhen-Xuan Li, Qi Liu, Hao-Ran Wang, Hao-Miao Li, \\ Hongwei Lv, Xiaochao Ma, Jianping Zhu, Yong-Kui Yu, Wen-Qun Xing; written on Henan Cancer \\ Hospital Thoracic Oncology Group (HCHTOG)
}

Department of Thoracic Surgery, The Affiliated Cancer Hospital of Zhengzhou University, Henan Cancer Hospital, Zhengzhou, China Correspondence to: Wen-Qun Xing, MD. Department of Thoracic Surgery, The Affiliated Cancer Hospital of Zhengzhou University, Henan Cancer Hospital, Zhengzhou 450008, China. Email: unique1vip@126.com.

Background: Neoadjuvant chemotherapy (NAC) and neoadjuvant chemoradiotherapy (NACR) are the
standard treatments for esophageal squamous cell carcinoma (ESCC). However, the 5-year overall survival
(OS) rate is still far from satisfactory. In recent years, immune checkpoint inhibitors (ICIs) have shown
promising results in the treatment of ESCC. More than 20 phase II clinical trials have been launched to
explore combinations of ICIs in the neoadjuvant setting for ESCC. Based on our phase II clinical trial, a
two-arm phase III trial was launched in Henan Cancer Hospital. ICIs combined with NAC may usher in a
new era and may benefit locally advanced, resectable ESCC patients. Methods: A two-arm phase III trial was launched in April 2020 in Henan Cancer Hospital. Patient recruitment will be completed within 18 months. The primary endpoint is event-free survival (EFS). The secondary endpoints include pathologic complete response (pCR), disease-free survival (DFS) rate, overall response rate (ORR), R0 resection rate, major pathologic response (MPR), adverse events (AEs), complication rate and quality of life (QOL). A biobank of pretreatment, resected tumor tissue and paired blood samples will be built for translational research in the future.

Discussion: This RCT directly compares NAC with neoadjuvant toripalimab plus chemotherapy in terms of EFS for locally advanced ESCC. The results may usher in a new era of resectable ESCC treatment.

Trial registration: NCT04280822 (https://www.clinicaltrials.gov/ct2/show/NCT04280822). Registered title: "A Phase III, Randomized Controlled Study of Neo-adjuvant Toripalimab (JS001) in Combination with Chemotherapy versus Neo-adjuvant Chemotherapy for Resectable Esophageal Squamous Cell Carcinoma". Version 1.0/Nov. 21, 2019.

Keywords: Esophageal cancer (EC); neoadjuvant immunochemotherapy; clinical trial; phase III

Submitted Jul 19, 2020. Accepted for publication Nov 20, 2020.

doi: $10.21037 /$ atm-20-5404

View this article at: http://dx.doi.org/10.21037/atm-20-5404

\footnotetext{
^ ORCID: 0000-0001-8196-7181.
} 


\section{Introduction}

Esophageal cancer (EC) is one of the most challenging aggressive malignancies. EC was the fifth leading cause of cancer death worldwide in 2018 (1). A series of multiinstitutional clinical trials from Western countries and Japan demonstrated that neoadjuvant chemoradiotherapy (NACR) improved the 5-year overall survival (OS) rate of resectable esophageal squamous cell carcinoma (ESCC) (2-4). However, the associated disadvantages of additional radiation cannot be neglected.

A long-awaited breakthrough in treatment has finally arrived (5). Cancer immunotherapy with multiple anti-PD-1 antibodies has been approved for 15 cancer types $(6,7)$. In 2019, several phase III randomized studies showed positive results when treating ESCC with anti-PD-1 antibodies (8). Observational studies in the neoadjuvant setting showed remarkably better response rates with pembrolizumab $(9,10)$ and nivolumab (11) than with chemotherapy alone. An increasing number of clinical trials have explored combinations of immune checkpoint inhibitors (ICIs) with neoadjuvant chemotherapy (NAC)/NACR (12).

ESCC has a diverse regional distribution $(13,14)$. Although, $53 \%$ of ESCC cases were from China (15), few randomized control trials (RCTs) have been performed in China. Thus, we are trying to perform RCTs to answer cutting-edge questions about ESCC. We have demonstrated the safety profiles of neoadjuvant immunochemotherapy with toripalimab plus taxol cis-platinum (TP) in our phase II study. Now, we are launching a phase III study to compare neoadjuvant immunochemotherapy with NAC. This may usher in a new chapter for the treatment of resectable ESCC and significantly prolong their 5-year survival. We present the following article in accordance with the SPIRIT reporting checklist (available at http://dx.doi.org/10.21037/atm-20-5404).

\section{Methods}

\section{Statement of ethical approval}

The Institutional Review Board of Henan Cancer Hospital approved the study, and the Ethics Committee of Henan Cancer Hospital gave ethical approval (ID: 2019092702). Signed informed consent will be obtained from all participants. The ethical principles of the Declaration of Helsinki will be strictly followed throughout the entire period of this trial (16). The authors are accountable for all aspects of the work in ensuring that questions related to the accuracy or integrity of any part of the work are appropriately investigated and resolved.

\section{Study design and setting}

This is a phase III, single-center, open label, randomized controlled clinical trial. Eligible patients will be randomized to arm A (paclitaxel $175 \mathrm{mg} / \mathrm{m}^{2} \mathrm{~d} 1$ + cisplatin $75 \mathrm{mg} / \mathrm{m}^{2}$ $\mathrm{d} 1+$ toripalimab $240 \mathrm{mg} \mathrm{d} 3$, every 3 weeks, 2 cycles) and arm B (paclitaxel $175 \mathrm{mg} / \mathrm{m}^{2} \mathrm{~d} 1$ + cisplatin $75 \mathrm{mg} / \mathrm{m}^{2} \mathrm{~d} 1$, every 3 weeks, 2 cycles) with a 1:1 allocation ratio and then undergo surgery (Figure 1). After surgery, there will be a maintenance period for arm A. In the maintenance period, toripalimab will be administered, $240 \mathrm{mg}$ ivgtt, d3, >30 min, 3 weeks (8 cycles at most).

\section{Study endpoints}

The primary outcome is event-free survival (EFS), which will be defined from the date of randomization to the date of the first record of unresectable disease, distant metastases, local recurrence from any cause, or death from any cause.

The secondary outcomes include the pathological complete response (pCR) rate, disease-free survival (DFS) rate, OS rate, overall response rate (ORR), R0 resection rate, major pathological response (MPR) rate, which will be defined as the percentage of patients who achieve a residual tumor $\leq 10 \%$, and adverse events (AEs) [as judged by the National Cancer Institute Common Terminology Criteria for Adverse Event, Version 5.0 (CTC AE5.0)]. The time frame for AEs will be up to 3 months after the last cycle of neoadjuvant treatment. The quality of life evaluation will include repeated assessments with the European Organization for the Research and Treatment of Cancer Quality of Life Questionnaire (EORTC QLQ)-C30, EORTC QLQ-OES18 and Nutrition Risk Screening 2002 (NRS-2002) (time frame: before surgery and 1, 3, 6, 9 months, and 1 year after the operation). The definition of postoperative complications were shown in Table S1.

\section{Participants}

In the Affiliated Cancer Hospital of Zhengzhou University/ Henan Cancer Hospital, once an esophageal disease patient is hospitalized, a physical examination was the first step. Then, esophageal endoscopic ultrasound, contrast-enhanced thoracic computed tomography (CT), cervical and abdominal ultrasound, esophagography, brain magnetic resonance imaging (MRI) and emission computed 


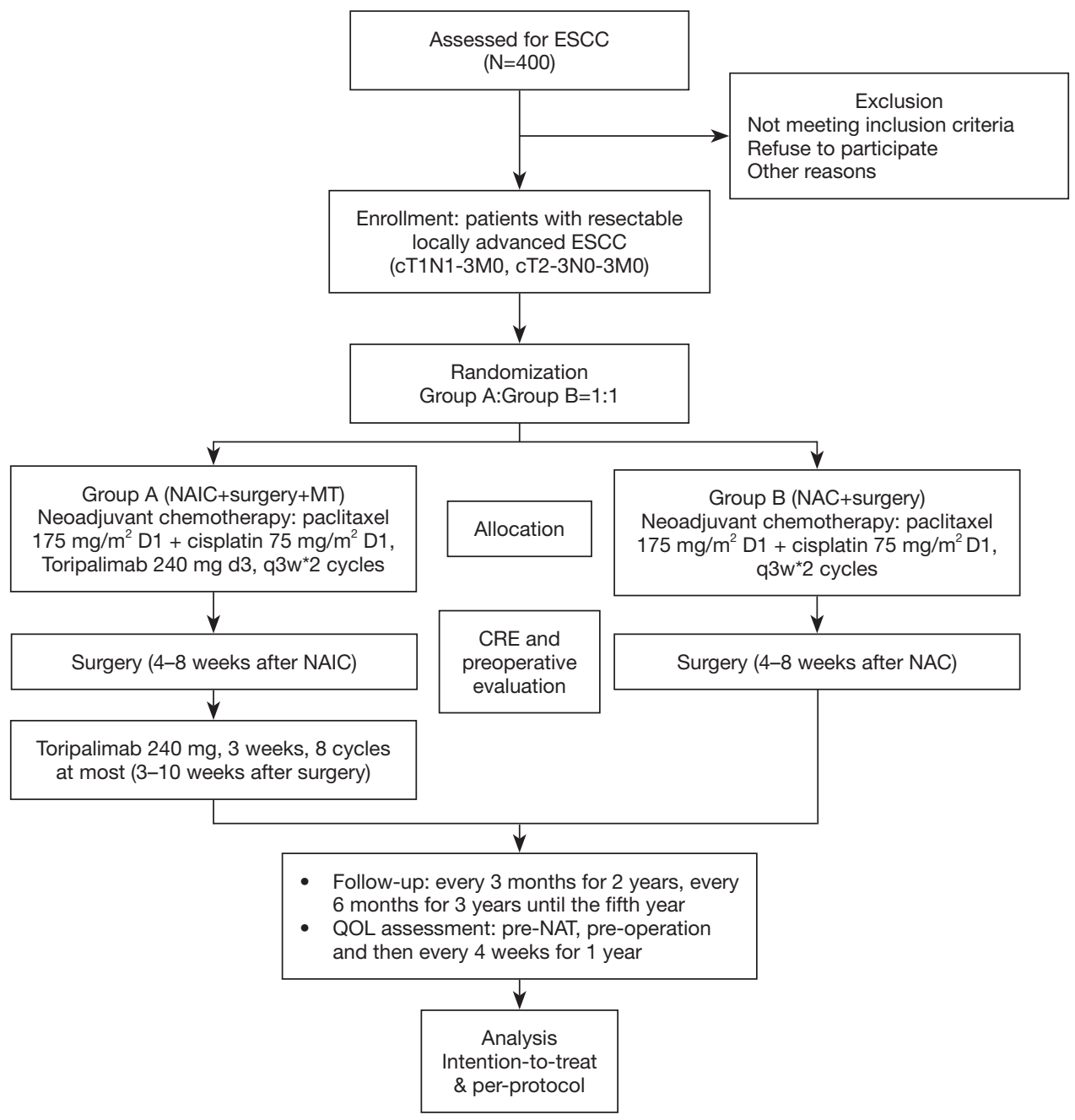

Figure 1 Flowchart of the study. ESCC, oesophageal squamous cell carcinoma; NAIC, neoadjuvant immunochemotherapy; MT, maintain treatment; NAC, neoadjuvant chemotherapy; CRE, clinical response evaluation; QOL, quality of life; NAT, neoadjuvant treatment.

tomography (ECT) are performed. If the patient wants, positron emission tomography-CT (PET/CT) can be performed instead of cervical and abdominal ultrasound and ECT. The multidisciplinary team (MDT) of the Thoracic Oncology Group discusses all the patients newly diagnosed with ESCC prior to any treatment. ESCC suitable for resection would be excised. Written informed consent was required from eligible patients before enrollment into the trial by principal investigators (PIs). Pretreatment staging was performed based on the 8th Union for International Cancer Control (UICC) TNM system (17).

\section{Inclusion criteria}

(I) Histologic diagnosis of locally advanced thoracic
ESCC staged as T1N1-3 or T2-3N0-3 M0 based on the UICC-TNM system;

(II) No metastatic lymph nodes found in the cervical region by color Doppler sonography;

(III) No prior anticancer therapy for EC;

(IV) Age between 18 and 75 years;

(V) No operative contraindications;

(VI) Absolute white blood cell count $\geq 4.0 \times 10^{9} / \mathrm{L}$, neutrophil $\geq 1.5 \times 10^{9} / \mathrm{L}$, platelets $\geq 100.0 \times 10^{9} / \mathrm{L}$, hemoglobin $\geq 90 \mathrm{~g} / \mathrm{L}$, normal liver and kidney function, total bilirubin (TBIL) $\leq 1.5 \mathrm{~N}$, aspartate aminotransferase (AST) $\leq 2.5 \mathrm{~N}$, alanine aminotransferase (ALT) $\leq 2.5 \mathrm{~N}$, prothrombin time $(\mathrm{PT}) \leq 1.5 \mathrm{~N}$, activated partial thromboplastin time 
(APTT) in the normal range, and endogenous creatinine clearance rate [clinical response evaluation (CRE) $] \leq 1.5$;

(VII) No diagnoses of other cancers except for prostatic cancer with at least 5 years of DFS and no prior anticancer therapy;

(VIII) Expected R0 resection.

(IX) Eastern Cooperative Oncology Group (ECOG) performance status of $0-1$.

(X) Contraceptive measures used during the study period and within 6 months of the end of the study, and non-lactating status for women;

(XI) Signed informed consent document on file.

\section{Exclusion criteria}

(I) Multiple primary cancers;

(II) Infectious diseases that require treatment;

(III) Requirement for continuous hormone treatment;

(IV) Unstable angina within 3 months and myocardial infarction within 6 months;

(V) Psychopathy;

(VI) Concomitant hemorrhagic disease;

(VII) Any unexpected reason preventing the patient from undergoing surgery;

(VIII) Inability to use gastric conduits after esophagectomy because of prior surgery;

(IX) Pregnant or breast feeding;

(X) Diagnosed or suspected allergies to cisplatin or paclitaxel;

(XI) Bronchial asthma requiring the intermittent use of bronchodilators or medical interventions;

(XII) Immunosuppressant usage due to coexisting diseases, with a dosage of more than $10 \mathrm{mg} /$ day orally and for more than 2 weeks before enrollment;

(XIII) Abnormal coagulation function [PT $>16 \mathrm{~s}$, APTT $>53 \mathrm{~s}$, thrombin time $(\mathrm{TT})>21 \mathrm{~s}$, fibrinogen (Fib) $<1.5 \mathrm{~g} / \mathrm{L}$ ], tendency to bleed or thrombolysis or anticoagulant therapy;

(XIV) Previous or present pulmonary fibrosis, interstitial pneumonia, pneumoconiosis, radiation pneumonia, or severely impaired lung function;

(XV) Autoimmune diseases, immune deficiency, or organ transplantation;

(XVI) Hepatitis B/C.

\section{Masking and randomization}

This study is unmasked. Once the recruitment criteria are met, the patients will be assigned (1:1) randomly by computer by research nurse. The stratification factors include lymph node status and PD-L1 expression. The randomization numbers will be set by a computer. The randomization will be conducted by a clinical research nurse. After patients sign the written consent form, they were randomized to arm A or arm B.

\section{Treatment regimens}

\section{Arm A (neoadjuvant immunochemotherapy arm,} toripalimab and chemotherapy)

Paclitaxel, $175 \mathrm{mg} / \mathrm{m}^{2}$, d1, cisplatin, $75 \mathrm{mg} / \mathrm{m}^{2}, \mathrm{~d} 1$, and toripalimab $240 \mathrm{mg} \mathrm{d} 3 ; 3$ weeks, 2 cycles. Three weeks to 2.5 months after surgery, the patients will receive toripalimab $240 \mathrm{mg} \mathrm{d} 3$ for a maximum of 3 weeks and 8 cycles.

\section{Arm B (NAC arm)}

Paclitaxel, $175 \mathrm{mg} / \mathrm{m}^{2}$, d1 , cisplatin, $75 \mathrm{mg} / \mathrm{m}^{2}$, d1; 3 weeks, 2 cycles.

The details of dose reductions and delays of chemotherapy/immunotherapy were specified in the protocol. If any type of Grade 4 toxicity based on CTCAE 5.0, National Cancer Institute Common Terminology Criteria for Adverse Events (version 5.0) happen, the dose of chemotherapy would be stopped or half dose. If immuno-related adverse events (irAE) happen, the dose of immunotherapy would be adjusted. From 4 weeks before randomization to the end of all treatment, any type of anticancer therapy which was not included in the protocol and vaccination was prohibited.

\section{Surgery}

Four weeks (8 weeks at most) after neoadjuvant therapy, surgery will be performed. A total 2-field lymphadenectomy will be strictly performed. Open thoracotomy esophagectomy, thoracoscopic esophagectomy and hybrid approaches are all acceptable.

\section{Follow-up}

The surveillance tests will include abdominal and cervical color Doppler ultrasonography and chest CT scans. All of these examinations will be conducted in the outpatient department. The frequency of the visits will be every 3 months during the first 2 years, every 6 months during 
the third to fifth years, and every year after the fifth year.

\section{Translational research}

Tissue and blood samples will be collected for translational research. Additional optional written consent for sample collection will be requested. The pretreatment biopsy tumor tissue, a postoperative biopsy of tumor tissue and paired blood samples will be collected. The biobank of HCHTOG1909 will built in the Henan Cancer Hospital Tissue Bank. All samples will be stored in liquid nitrogen.

\section{Statistical analysis}

The sample size calculation is based on the EFS. We assumed that 238 patients who achieved EFS will be collected 46 months after the first randomized patient. The two-sided type I error was set as $5 \%$, with a power of $90 \%$ and a $10 \%$ drop out rate in the first 5 years. The estimated enrollment period was 1.5 years. EFS is exponentially distributed. We assumed that the hazard ratio (HR) in both groups was 0.68 and that the median EFS duration was 20 months. A total of 400 patients (200 patients in each group) will be enrolled in this trial.

The primary endpoint of EFS will be calculated by comparing toripalimab plus chemotherapy with chemotherapy alone using a log-rank test. The Cox regression model will be used to estimate HRs. The KaplanMeier method will be adopted to estimate the event rates over time within each group. The efficacy and safety results will be monitored by an external data committee to decide if the trial will continue. The safety of randomized patients who received at least one dose of the study medication will be analyzed.

The first interim analysis is scheduled to be 1 year after the last randomized patients. The pCR rate will be analyzed. The second interim analysis is planned for when $75 \%$ EFS (211 patients) is achieved. The EFS will be reported. Sixty months after the last patient is recruited, OS will be analyzed. The intention-to-treat (ITT) principle will be followed. All randomized patients will be analyzed. The patients who comply with the protocol will be collected for the per-protocol analysis, a secondary analysis. This analysis will be used to supplement the ITT analysis.

\section{Monitoring}

The Department of Clinical Research Management, Good
Clinical Practice (GCP) and Ethics Committee of the Affiliated Cancer Hospital of Zhengzhou University/Henan Cancer Hospital will evaluate the study progress, data integrity and patient safety. The trial data will be reviewed every 6 months by the clinical research management department. The PI will be identifiable in the final trial database. The data was collected by research nurse with Electronic Data Capture (EDC) (linkdoc company). The trial will be independent from the sponsor. A medical insurance will cover the compensation to those who suffer harm from trial participation.

\section{Participating institutions}

Henan Cancer Hospital/The Affiliated Cancer Hospital of ZhengZhou University. PI: Jiangong Zhang, MD \& PhD \& Yan Zheng, MD \& PhD (sunnyzheng1@126.com).

\section{Funding, registration and current status}

This is an investigator initial trial. It is mainly sponsored by Henan Cancer Hospital and PIs. Currently, this work was partly supported by the Henan province health and Family Planning Commission (grant number 2018020497, 2018), Henan Anti-Cancer Association the Youth Talent Project (grant number 2019HYTP018, 2019), and by the Wu Jieping Medical Fund (grant numbers 320.6799.15062, 2015 and 320.2730.1892, 2018). Before recruitment began, this trial was registered at ClinicalTrials.gov on February 21, 2020 (registration number: NCT04280822). The trial is currently in patient recruitment, which began in April 2020.

\section{Discussion}

In the past 30 years, a series of multicenter clinical trials have confirmed that the standard treatments for resectable ESCC are NAC and NACR $(2,3,18)$. Based on the CROSS (3) and NEOCRTEC5010 (4) studies, NACR showed strong evidence of better survival. However, according to the FFCD9901 trial, complication of treatments cannot be neglected (19). The postoperative complications of lung infection and anastomotic leakage are critical limitations. There could also be technical difficulties for future surgeries after radiation (20). In the real world, NARC is not accepted by most surgeons in China. Based on evidence from the 9907 trial, NAC with acceptable complementary efforts is the standard treatment in China and Japan. Without direct comparisons in clinical trials, we 
cannot say which treatment better for prolonging the OS of patients with ESCC. To date, the best OS was achieved with NACR. It remains unclear whether NAC or NACR leads to better survival. However, NAC has much lower complication rates (21).

In 2014, pembrolizumab, the first ICI, was approved by the FDA for unresectable melanoma (22). ICIs have shown promising anticancer activity. The evaluation of ICIs in solid tumors, especially in ESCC, yielded positive results in phase III clinical studies $(23,24)$. Even after NAC (25), approximately $50 \%$ of patients with resectable ESCC relapsed within 1 year after surgery (26). Following encouraging conclusions in the metastatic setting, ICIs have already been investigated for localized ESCC. We anticipate that neoadjuvant ICIs plus chemotherapy may usher in a new era of ESCC treatment. The improved OS and acceptable toxicity of the combination of chemotherapy and ICIs was fully demonstrated in the Keynote 180 (23) and 181 trials (24). Nivolumab plus NACR for esophageal/ gastroesophageal junction (GEJ) cancer (27) has already been investigated in a phase $\mathrm{Ib}$ study. There were no unexpected AEs. Nivolumab alone or with ipilimumab in combination with NACR for esophageal and GEJ adenocarcinoma was investigated in a phase II/III trial (NCT03604991). The pCR rate was the primary endpoint, and the secondary endpoint was DFS (28). Our phase II study (NCT03985670), "Neoadjuvant Toripalimab Plus Chemotherapy in Local Advanced Esophageal Squamous Cell Carcinoma", also demonstrated the safety of this combined treatment for resectable ESCC.

The sequence of ICIs and chemotherapy is under debate. Some studies have suggested ICIs should be administered after chemotherapy $(29,30)$. Some studies have reported that ICIs administered before chemotherapy may lead to a better response. If ICIs are given first, they may preserve the immune cells. Otherwise, chemotherapy may result in immunosuppression and myelosuppression and affect the efforts of ICIs (31). In our previous phase II study, different sequences of toripalimab and chemotherapy were explored. Our initial results showed that compared with toripalimab and chemotherapy administered on the same day, toripalimab administered after chemotherapy led to a significantly better clinical response [based on chest CT scans assessed with the Response Evaluation Criteria in Solid Tumors (RECIST) 1.1] and a trend towards a better pathological response [tumor regression grade (TRG)]. This mechanism needs to be further studied. Our phase III study will administer chemotherapy on the first day and then toripalimab on day 3 .

The clinical trials of ICIs have occurred at a breathtaking pace. The time between phase I studies and phase III studies has shortened (32). More than 20 studies on immunotherapy in the perioperative setting for resectable EC were registered on clinicaltrials.gov. Henan Province has the largest number of diagnosed ESCC cases in China. This large-scale phase III clinical study may be easily and quickly completed in the Henan Cancer Hospital (33). Our phase II clinical trial showed promising results with neoadjuvant toripalimab plus chemotherapy for ESCC. Therefore, a phase III study was launched to directly compare NAC versus neoadjuvant immunochemotherapy for locally advanced ESCC.

\section{Conclusions}

ICIs have demonstrated strong anticancer effects in combination with chemotherapy for advanced ESCC. Our phase II clinical study demonstrated the primary safety and efficacy of toripalimab plus chemotherapy for resectable ESCC. A strong rationale exists to further evaluate the role of toripalimab plus chemotherapy in the neoadjuvant setting for patients with resectable ESCC. Herein, we described the methodology of the HCHTOG1909 study. Hopefully, the results will start usher in a new era of ESCC treatment.

\section{Acknowledgments}

Funding: This work was supported by the Henan Province Health and Family Planning Commission (grant number 2018020497, 2018), Henan Anti-Cancer Association the Youth Talent Project (grant number 2019HYTP018, 2019), and by the Wu Jieping Medical Fund (grant numbers 320.6799.15062, 2015 and 320.2730.1892, 2018).

\section{Footnote}

Reporting Checklist: The authors have completed the SPIRIT reporting checklist. Available at http://dx.doi.org/10.21037/ atm-20-5404

Conflicts of Interest: All authors have completed the ICMJE uniform disclosure form (available at http://dx.doi. org/10.21037/atm-20-5404). The authors have no conflicts of interest to declare.

Ethical Statement: The authors are accountable for all 
aspects of the work in ensuring that questions related to the accuracy or integrity of any part of the work are appropriately investigated and resolved. The Institutional Review Board of Henan Cancer Hospital approved the study, and the Ethics Committee of Henan Cancer Hospital gave ethical approval (ID: 2019092702). Signed informed consent will be obtained from all participants. The ethical principles of the Declaration of Helsinki (as revised in 2013) will be strictly followed throughout the entire period of this trial.

Disclaimer: The TopAlliance offered the Toripalimab for free for this study.

Open Access Statement: This is an Open Access article distributed in accordance with the Creative Commons Attribution-NonCommercial-NoDerivs 4.0 International License (CC BY-NC-ND 4.0), which permits the noncommercial replication and distribution of the article with the strict proviso that no changes or edits are made and the original work is properly cited (including links to both the formal publication through the relevant DOI and the license). See: https://creativecommons.org/licenses/by-nc-nd/4.0/.

\section{References}

1. Bray F, Ferlay J, Soerjomataram I, et al. Global cancer statistics 2018: GLOBOCAN estimates of incidence and mortality worldwide for 36 cancers in 185 countries. CA Cancer J Clin 2018;68:394-424.

2. Zheng Y, Li Y, Liu X, et al. Reevaluation of neoadjuvant chemotherapy for esophageal squamous cell carcinoma: A meta-analysis of randomized controlled trials over the past 20 years. Medicine (Baltimore) 2015;94:e1102.

3. van Heijl M, van Lanschot JJ, Koppert LB, et al. Neoadjuvant chemoradiation followed by surgery versus surgery alone for patients with adenocarcinoma or squamous cell carcinoma of the esophagus (CROSS). BMC Surg 2008;8:21.

4. Yang H, Liu H, Chen Y, et al. Neoadjuvant chemoradiotherapy followed by surgery versus surgery alone for locally advanced squamous cell carcinoma of the esophagus (NEOCRTEC5010): A phase III multicenter, randomized, open-label clinical trial. J Clin Oncol 2018;36:2796-803.

5. Kuo HY, Guo JC, Hsu CH. Anti-PD-1 immunotherapy in advanced esophageal squamous cell carcinoma: A longawaited breakthrough finally arrives. J Formos Med Assoc
2020;119:565-8.

6. Kaplan DK, Whyte RI, Donnelly RJ.

Oesophagogastrectomy using stapling instruments. Eur J Cardiothorac Surg 1988;2:95-9.

7. Available online: https://www.accessdata.fda.gov/ drugsatfda_docs/label/2019/125514s065lbl.pdf [cited 2019 Sept 7].

8. Zayac A, Almhanna K. Esophageal, gastric cancer and immunotherapy: Small steps in the right direction? Transl Gastroenterol Hepatol 2020;5:9.

9. Van Schil PE. Reply to "Pulmonary metastasectomy: Where is the evidence?": Absence of evidence is not evidence of absence! J Thorac Oncol 2015;10:e14-5.

10. Doi T, Piha-Paul SA, Jalal SI, et al. Updated results for the advanced esophageal carcinoma cohort of the phase Ib KEYNOTE-028 study of pembrolizumab (MK-3475). J Clin Oncol 2016;34:abstr 7.

11. Kudo T, Hamamoto Y, Kato K, et al. Nivolumab treatment for oesophageal squamous-cell carcinoma: An open-label, multicentre, phase 2 trial. Lancet Oncol 2017;18:631-9.

12. Huang TX, Fu L. The immune landscape of esophageal cancer. Cancer Commun (Lond) 2019;39:79.

13. Somdyala NI, Bradshaw D, Gelderblom WC, et al. Cancer incidence in a rural population of South Africa, 1998-2002. Int J Cancer 2010;127:2420-9.

14. Lin Y, Totsuka Y, He Y, et al. Epidemiology of esophageal cancer in Japan and China. J Epidemiol 2013;23:233-42.

15. Siegel R, Desantis C, Jemal A. Colorectal cancer statistics, 2014. CA Cancer J Clin 2014;64:104-17.

16. World Medical Association I. Declaration of Helsinki. Ethical principles for medical research involving human subjects. J Indian Med Assoc 2009;107:403-5.

17. Rice TW, Ishwaran H, Ferguson MK, et al. Cancer of the esophagus and esophagogastric junction: An eighth edition staging primer. J Thorac Oncol 2017;12:36-42.

18. Lordick F, Mariette C, Haustermans K, et al. Oesophageal cancer: ESMO Clinical Practice Guidelines for diagnosis, treatment and follow-up. Ann Oncol 2016;27:v50-7.

19. Mariette C, Dahan L, Mornex F, et al. Surgery alone versus chemoradiotherapy followed by surgery for stage I and II esophageal cancer: Final analysis of randomized controlled phase III trial FFCD 9901. J Clin Oncol 2014;32:2416-22.

20. Ashok A, Tiwari V, Jiwnani S, et al. Controversies in preoperative therapy in esophageal cancer: Current evidence and ongoing research. Ann Gastroenterol Surg 2019;3:592-7.

21. Sun HB, Xing WQ, Liu XB, et al. Neoadjuvant 
chemotherapy versus neoadjuvant chemoradiotherapy for locally advanced oesophageal squamous cell carcinoma: A single-Centre, open-label, randomized, controlled, clinical trial (HCHTOG1903). BMC Cancer 2020;20:303.

22. Robert C, Ribas A, Wolchok JD, et al. Anti-programmeddeath-receptor-1 treatment with pembrolizumab in ipilimumab-refractory advanced melanoma: A randomised dose-comparison cohort of a phase 1 trial. Lancet 2014;384:1109-17.

23. Shah MA, Kojima T, Hochhauser D, et al. Efficacy and safety of pembrolizumab for heavily pretreated patients with advanced, metastatic adenocarcinoma or squamous cell carcinoma of the esophagus: The phase 2 KEYNOTE-180 study. JAMA Oncol 2019;5:546-50.

24. Kato K, Shah MA, Enzinger P, et al. KEYNOTE-590: Phase III study of first-line chemotherapy with or without pembrolizumab for advanced esophageal cancer. Future Oncol 2019;15:1057-66.

25. van Hagen $\mathrm{P}$, Hulshof MC, van Lanschot JJ, et al. Preoperative chemoradiotherapy for esophageal or junctional cancer. N Engl J Med 2012;366:2074-84.

26. Kesler KA, Helft PR, Werner EA, et al. A retrospective analysis of locally advanced esophageal cancer patients treated with neoadjuvant chemoradiation therapy followed by surgery or surgery alone. Ann Thorac Surg 2005;79:1116-21.

27. Meindl-Beinker NM, Betge J, Gutting T, et al. A

Cite this article as: Zheng $\mathrm{Y}$, Liu XB, Sun HB, Xu J, Shen S, Ba YF, Yan M, Qin Z, Liu BX, Wang ZF, Liu SL, Zhang RX, Chen PN, Liang GH, Yuan D, Li ZX, Liu Q, Wang HR, Li HM, Lv H, Ma X, Zhu J, Yu YK, Xing WQ; written on Henan Cancer Hospital Thoracic Oncology Group (HCHTOG). A phase III study on neoadjuvant chemotherapy versus neoadjuvant toripalimab plus chemotherapy for locally advanced esophageal squamous cell carcinoma: Henan Cancer Hospital Thoracic Oncology Group 1909 (HCHTOG1909). Ann Transl Med 2021;9(1):73. doi: 10.21037/atm-20-5404 multicenter open-label phase II trial to evaluate nivolumab and ipilimumab for 2nd line therapy in elderly patients with advanced esophageal squamous cell cancer (RAMONA). BMC Cancer 2019;19:231.

28. Vivaldi C, Catanese S, Massa V, et al. Immune checkpoint inhibitors in esophageal cancers: Are we finally finding the right path in the mist? Int J Mol Sci 2020;21:1658.

29. Huang AC, Postow MA, Orlowski RJ, et al. T-cell invigoration to tumour burden ratio associated with antiPD-1 response. Nature 2017;545:60-5.

30. Yamasaki M, Funaishi K, Kawamoto K, et al. Platinumdoublet chemotherapy followed by pembrolizumab therapy for lung cancer with lymphangitis carcinomatosa mimicking interstitial pneumonitis: A case report. Medicine (Baltimore) 2019;98:e16834.

31. Ramchandren R, Domingo-Domenech E, Rueda A, et al. Nivolumab for newly diagnosed advanced-stage classic hodgkin lymphoma: Safety and efficacy in the phase II checkmate 205 study. J Clin Oncol 2019;37:1997-2007.

32. Ku GY. The current status of immunotherapies in esophagogastric cancer. Hematol Oncol Clin North Am 2019;33:323-38.

33. Zheng Y, Li Y, Liu X, et al. A phase III, multicenter randomized controlled trial of neo-adjuvant chemotherapy paclitaxel plus cisplatin versus surgery alone for stage IIAIIIB esophageal squamous cell carcinoma. J Thorac Dis 2017;9:200-4. 
Table S1 Definition of complications

\begin{tabular}{|c|c|}
\hline Complication & Criteria \\
\hline \multicolumn{2}{|l|}{ Cardiac complications } \\
\hline Cardiac infarction & Confirmed by electrocardiography or echocardiography and cardiac enzyme monitoring \\
\hline Heart failure & Confirmed by echocardiography or necessitating pressure agents \\
\hline Arrhythmia & ECG confirmed and necessitating medication \\
\hline Pericarditis & Diagnosed by pericardiocentesis and requiring treatment \\
\hline Cardiac tamponade & Diagnosed by echocardiography and requiring treatment \\
\hline \multicolumn{2}{|l|}{ Respiratory complications } \\
\hline Pneumonia & X-ray or CT confirmed and necessitating antibiotic treatment \\
\hline Airway necrosis or fistulae & Confirmed by endoscopy \\
\hline Respiratory failure & $\begin{array}{l}\text { Need for mechanical ventilation for greater than } 24 \mathrm{~h} \text { in a patient who requires reintubation after } \\
\text { surgery OR need for mechanical ventilation for greater than } 72 \mathrm{~h} \text { in a patient who is not extubated on } \\
\text { the day of surgery (34) }\end{array}$ \\
\hline ARDS & Berlin definition (35) \\
\hline Atelectasis & X-ray or CT confirmed and requiring bronchoscopy \\
\hline Air leak & Chest tube maintenance for air leak for $>7$ days postoperatively \\
\hline Pulmonary embolus & Confirmed by angio-CT scan \\
\hline Pleural effusions & X-ray or CT confirmed and requiring treatment \\
\hline Chest infection & Supported by positive bacterial culture \\
\hline Pneumothorax & X-ray or CT confirmed and requiring treatment \\
\hline Mediastinitis & Supported by positive bacterial culture \\
\hline \multicolumn{2}{|l|}{ Gastrointestinal complications } \\
\hline Anastomotic leak & $\begin{array}{l}\text { Extravasation of water-soluble contrast during a swallow study, visualization of either anastomotic } \\
\text { dehiscence or fistulae during endoscopy or visible loss of saliva or methylene blue through the } \\
\text { cervical wound }\end{array}$ \\
\hline Nonanastomotic leak & Radiologically or endoscopically identified \\
\hline Conduit necrosis & Endoscopically or intraoperatively identified \\
\hline Intra-abdominal abscess & Supported by positive bacterial culture \\
\hline Peritoneal effusion & Doppler ultrasound or CT confirmed and requiring treatment \\
\hline Gastrointestinal bleeding & Requiring intervention or transfusion \\
\hline Liver failure & $\begin{array}{l}\text { Need for FFP to correct INR in patient with serum bilirubin }>12 \mathrm{mg} / \mathrm{dL} \text { or INR }>2.5 \text { in a patient with } \\
\text { serum bilirubin }>12 \mathrm{mg} / \mathrm{dL}\end{array}$ \\
\hline Intestinal obstruction & Clinical symptoms, radiological confirmation and needing treatment \\
\hline Delayed gastric emptying & Clinical symptoms, radiological confirmation and needing treatment \\
\hline Diaphragmatic hernia & Diagnosed by barium swallow or CT scan and then confirmed intraoperatively \\
\hline \multicolumn{2}{|l|}{ Other complication } \\
\hline Transient ischemic attack & Defined according to American Heart Association/American Stroke Association expert consensus \\
\hline Cerebral infarction & CT or MRI confirmed \\
\hline Cerebral hemorrhage & CT or MRI confirmed \\
\hline Urinary tract infection & $\begin{array}{l}\text { Presence of microorganisms in the urine accompanied by one or more of the following: dysuria, } \\
\text { urgency, loin pain, tenderness, pyrexia or pyuria }\end{array}$ \\
\hline Urinary retention & Requiring reinsertion of urinary catheter \\
\hline Acute renal insufficiency & Doubling of baseline creatinine \\
\hline Renal failure & Need for dialysis in a patient not on dialysis preoperatively \\
\hline Wound infection & Requiring opening of wound or antibiotics \\
\hline Bacteremia & Supported by at least one blood culture positive for pathogenic organisms \\
\hline Sepsis & $\begin{array}{l}\text { Temperature }>38 \text { or }<36^{\circ} \mathrm{C} \text {, heart rate }>90 \text { beats } / \mathrm{min} \text {, WBC count }>12,000 \text { cells } / \mathrm{mL} \text { or } \\
<4,000 \text { cells } / \mathrm{mL} \text {, and bacteremia }\end{array}$ \\
\hline Postoperative hemorrhage & Postoperative bleeding $>2,000 \mathrm{~mL}$ or need for reoperation \\
\hline Recurrent laryngeal nerve paralysis & Identified by endoscopic examination \\
\hline Chylothorax & $\begin{array}{l}\text { Increase in chest tube output with enteral alimentation, change in the nature of the output to a milky } \\
\text { appearance, and confirmation by a physical-chemical analysis of the fluid }\end{array}$ \\
\hline Deep venous thrombosis & Clinical symptoms and vascular color echo-Doppler diagnosis \\
\hline Fat necrosis & Clinical symptoms and exception of infection \\
\hline Subcutaneous effusion & Clinical symptoms and requiring treatment \\
\hline
\end{tabular}

ECG, electrocardiogram; CT, computed tomography; ARDS, acute respiratory distress syndrome; FFP, fresh frozen plasma; INR, international normalized ratio; MRI, magnetic resonance imaging.

\section{References}

34. Strasberg SM, Linehan DC, Hawkins WG. The accordion severity grading system of surgical complications. Ann Surg 2009;250:177-86

35. ARDS Definition Task Force; Ranieri VM, Rubenfeld GD, et al. Acute respiratory distress syndrome: the Berlin Definition. JAMA 2012;307:2526-33. 